\title{
A cold-gas reservoir to fuel the M 31 nuclear black hole and stellar cluster ${ }^{\star}$
}

\author{
A.-L. Melchior ${ }^{1,2}$ and F. Combes ${ }^{1}$ \\ 1 LERMA, Observatoire de Paris, UMR 8112, 61 avenue de l'Observatoire, 75014 Paris, France \\ e-mail: [A.L.Melchior;Francoise.Combes]@obspm.fr \\ 2 Université Pierre et Marie Curie-Paris 6, 4 place Jussieu, 75252 Paris Cedex 05, France
}

Received 10 August 2012 / Accepted 12 October 2012

\begin{abstract}
With IRAM-30 m/HERA, we have detected CO(2-1) gas complexes within $30 \operatorname{arcsec}(\sim 100 \mathrm{pc})$ from the center of M31 that amount to a minimum total mass of $4.2 \times 10^{4} M_{\odot}$ (one third of the positions are detected). Averaging the whole HERA field, we show that there is no additional undetected diffuse component. Moreover, the gas detection is associated with gas lying on the far side of the M 31 center as no extinction is observed in the optical, but some emission is present on infrared Spitzer maps. The kinematics is complex. (1) The velocity pattern is mainly redshifted: the dynamical center of the gas differs from the black hole position and the maximum of optical emission, and only the redshifted side is seen in our data. (2) Several velocity components are detected in some lines of sight. Our interpretation is supported by the reanalysis of the effect of dust on a complete planetary nebula sample. Two dust components are detected with respective position angles of $37 \mathrm{deg}$ and $-66 \mathrm{deg}$. This is compatible with a scenario where the superposition of the $(\mathrm{PA}=37 \mathrm{deg})$ disk is dominated by the $10 \mathrm{kpc}$ ring and the inner $0.7 \mathrm{kpc}$ ring detected in infrared data, whose position angle (-66 deg) we measured for the first time. The large-scale disk, which dominates the HI data, is steeply inclined $(i=77 \mathrm{deg}$ ), warped and superposed on the line of sight on the less inclined inner ring. The detected $\mathrm{CO}$ emission might come from both components.
\end{abstract}

Key words. galaxies: individual: M 31 - galaxies: nuclei - galaxies: kinematics and dynamics - galaxies: bulges - galaxies: ISM ISM: molecules

\section{Introduction}

M31 is usually described as a quiescent galaxy with little star formation, at a level of $0.4 M_{\odot} \mathrm{yr}^{-1}$ (e.g. Barmby et al. 2006; Tabatabaei \& Berkhuijsen 2010; Azimlu et al. 2011) and with ultra-weak nuclear activity (del Burgo et al. 2000). The presence of a very massive black hole (Dressler 1984) and the lack of gas within 300 pc (Nieten et al. 2006; Chemin et al. 2009; Braun et al. 2009) suggest that the main gas reservoir has been accreted and is exhausted, although some gas is detected within $1 \mathrm{kpc}$ from the center (Melchior et al. 2000; Melchior \& Combes 2011). From optical emission lines Jacoby et al. (1985) estimated an ionized gas mass on the order of $1500 M_{\odot}$, which can be accounted for by mass loss from evolving stars. Groves et al. (2012) relied on Herschel data to argue that the dust properties are well accounted for by the stellar heating. Small amounts of molecular gas have been detected in directions more than $300 \mathrm{pc}$ from the center. These can be associated with dust features in this area (Melchior et al. 2000; Melchior \& Combes 2011). While the center of M 31 hosts a supermassive black hole with a mass of 0.7-1.4 $\times 10^{8} M_{\odot}($ Bacon et al. 2001; Bender et al. 2005), it is one of the most silent ones (Garcia et al. 2010), although beginning in 2008 it started to murmur (Li et al. 2011). Furthermore, it exhibits many coherent structures that are interpreted as tracers of its merging history: there is a lopsided nuclear disk (Lauer et al. 1993) with two stellar components, P1 and P2 separated by $0.45^{\prime \prime}$ in the center. From the kinematics, the black hole is located in between P1 and P2, but closer to P2. An A-star cluster

\footnotetext{
* The reduced spectra (FITS files) are only available at the CDS via anonymous ftp to cdsarc.u-strasbg. fr (130.79.128.5) or via http://cdsarc.u-strasbg.fr/viz-bin/qcat?]/A+A/549/A27
}

(see also Kormendy \& Bender 1999), detected in a third component (P3) of M31's double nucleus by Bender et al. (2005), can be associated with a recent star formation episode. This occurred $200 \mathrm{Myr}$ ago, involved a total mass in the range $10^{4}-10^{6} M_{\odot}$, and corresponds to an accretion rate of $10^{-4}-10^{-2} M_{\odot} \mathrm{yr}^{-1}$. Its presence so close to the black hole raises a number of questions: how were young stars formed deeply inside to the tidal field of a supermassive black hole and how these stars have formed while there is no cold gas detected in the surroundings (e.g. Lauer et al. 2012; Li et al. 2009). In the Galaxy, SgrA* has experienced X-ray flares, attributed to the infall of gas, while a cloud of gas identified by Gillessen et al. (2012) is expected to fall onto the black hole in 2013. M 31* is experiencing a similar murmur according to Li et al. (2011), suggesting some gas infall.

In addition to the young star cluster, an ionized gas outflow was detected in X-rays along the minor axis of the galaxy by Bogdán \& Gilfanov (2008), perpendicular to the main disk. The relative intensity of the outflow on both sides is compatible with the intensity of the observed B extinction: the NW side is more extinguished than the SE side. As discussed in Melchior \& Combes (2011), the velocity field of the circumnuclear region $\left(40^{\prime \prime} \times 40^{\prime \prime}\right.$ or $\left.150 \mathrm{pc} \times 150 \mathrm{pc}\right)$ measured in optical ionized gas does not exhibit any clear rotation pattern: apart from a spot at the systemic velocity in M31's center, the whole area is blueshifted with respect to the systemic velocity. This coherent flow of ionized gas is decoupled from the stellar kinematics (Bender et al. 2005; del Burgo et al. 2000; Saglia et al. 2010), and could be connected to the recent star formation activity. Throughout the paper we assume a distance to M 31 of $780 \mathrm{kpc}$ (Vilardell et al. 2006), i.e. 1 arcsec $=3.8 \mathrm{pc}$. Most up-to-date results, based on cepheids, quote $752 \pm 27 \mathrm{kpc}$ 


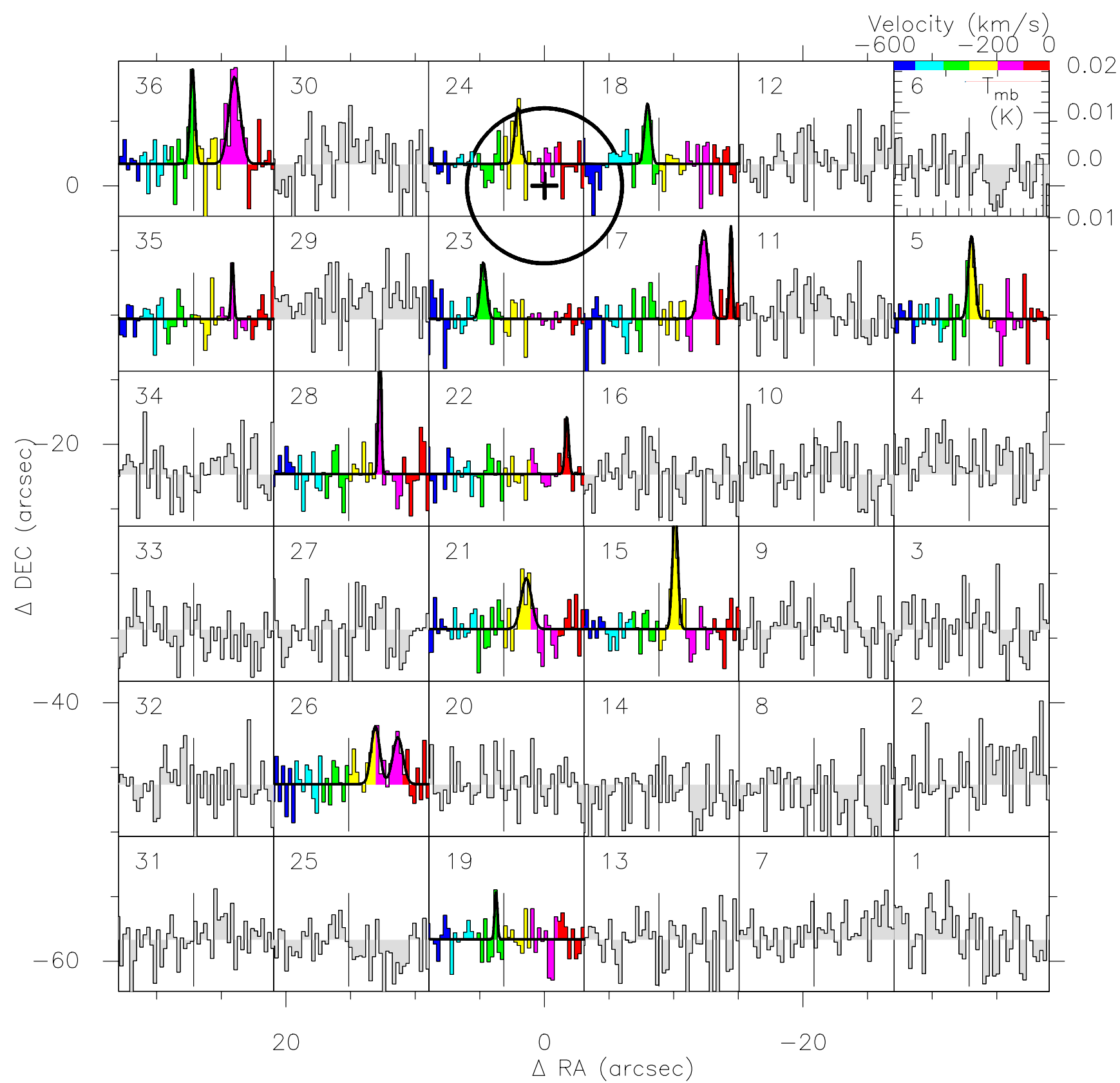

Fig. 1. Field M 31-1a centered on RA: 00:42:44.1 and Dec: $+41: 15: 42$ (J2000) observed with IRAM-30 m/HERA. The center is indicated with a cross and the circle displays the $12^{\prime \prime}$ beam. The $Y$ scale of each spectrum is in main-beam temperature $\left(T_{\mathrm{mb}}\right.$ between -0.01 and $0.02 \mathrm{mK}$, as indicated on the top right corner), while the $X$-axis shows the velocity for the range between -600 and $0 \mathrm{~km} \mathrm{~s}^{-1}$ smoothed to $13 \mathrm{~km} \mathrm{~s}^{-1}$ velocity resolution. A thin line indicates the systemic velocity at $-310 \mathrm{~km} \mathrm{~s}^{-1}$. The color-coding (displayed in the top right corner) of the velocity is used for the spectra with $3 \sigma$ detections.

(Riess et al. 2012). However, for coherence with previous works we keep $780 \mathrm{kpc}$, which lies within $1 \sigma$ uncertainties of these new results.

Some cold gas is expected to feed the black hole, even though in contrast with the Milky Way, a general lack of HI in the vicinity of M31's nucleus has been noted for several decades (Emerson 1976; Bajaja \& Shane 1982). In this paper, we present the first molecular detections within 30 arcsec from the center. In Sect. 2, we present the new observations performed at IRAM-30 m near the center of M 31. In Sect. 3.1, we analyze our molecular gas detections and compare them with other wavelengths. In Sect. 4, we discuss the interpretation of these data.

\section{Observations}

In the period between November 2011 and March 2012, we used the $1.3 \mathrm{~mm}$ multibeam HEterodyne Receiver Array (HERA) at the IRAM-30 m telescope (Schuster et al. 2004) to conduct a $\mathrm{CO}(2-1)$ survey of M31's $0.7 \mathrm{kpc}$ inner ring. One of the fields of this survey contained the center of M31. We refer to this field as M31-1a and show a $60^{\prime \prime} \times 60^{\prime \prime}$ map with $12^{\prime \prime}$ angular resolution in Figs. 1 and 2 . We thus created a $60^{\prime \prime}$ by $60^{\prime \prime}$ map with $12^{\prime \prime}$ spatial resolution for the $\mathrm{CO}(2-1)$ line. Data were acquired in wobbler-switching mode, using the Wideband Line Multiple Autocorrelator (WILMA) facility as backend. The 

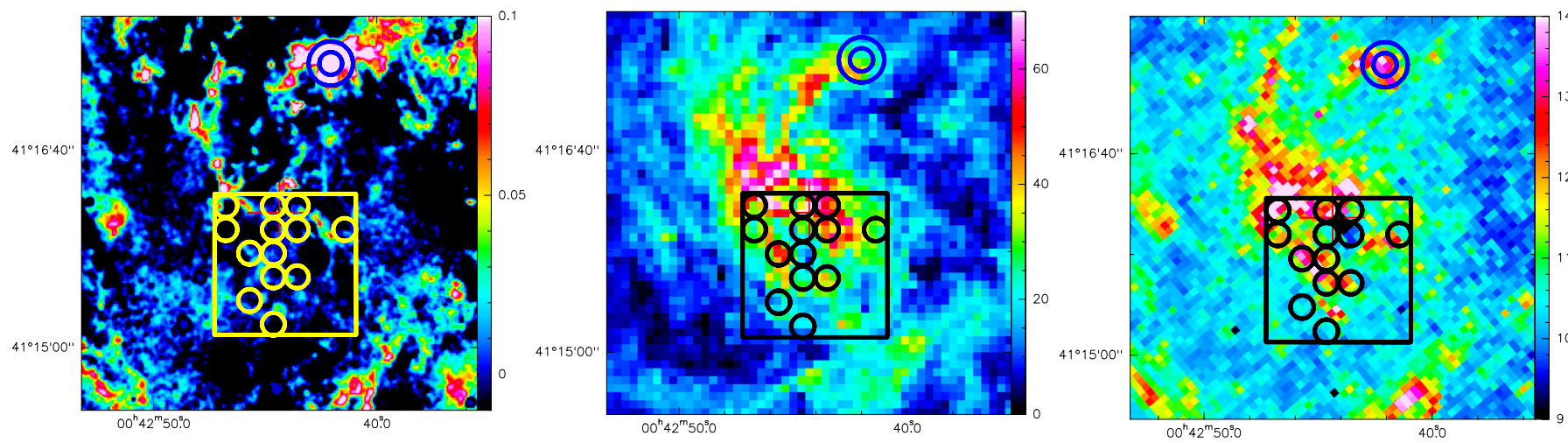

Fig. 2. Field M31-1a centered on RA: 00:42:44.1 and Dec: +41:15:42 (J2000) observed at IRAM-30 m HERA, superimposed on the A $A_{\text {observed }}^{B}$ observed extinction map (Melchior et al. 2000, left panel), H $\alpha$ and [NII] emission map (Ciardullo et al. 1988, middle panel), and $8 \mu$ m Spitzer emission map (Block et al. 2006, right panel). The circles correspond to the $\geq 3 \sigma$ detections discussed in this paper.

Table 1. Log of observations.

\begin{tabular}{lccl}
\hline \hline Date & $\left\langle T_{\text {sys }}\right\rangle$ & $t_{\text {integration }}$ & $\#$ pixels (scans) \\
\hline 2011-Nov-08 & $320 \mathrm{~K}$ & $276 \mathrm{~min}$ & $36(144)$ \\
$2011-$ Nov-10 & $411 \mathrm{~K}$ & $276 \mathrm{~min}$ & $36(72)$ \\
$2011-$ Nov-27 & $274 \mathrm{~K}$ & $276 \mathrm{~min}$ & $18(36)$ \\
$2012-$ Feb-12 & $251 \mathrm{~K}$ & $144 \mathrm{~min}$ & $36(144)$ \\
$2012-F e b-24$ & $318 \mathrm{~K}$ & $300 \mathrm{~min}$ & $36(72)$ \\
2012-Mar-11 & $351 \mathrm{~K}$ & $295 \mathrm{~min}$ & $36(72)$ \\
\hline
\end{tabular}

wobbler throw (ranging between $60^{\prime \prime}$ to $210^{\prime \prime}$ in azimuth) was adjusted every $30 \mathrm{~min}$ to avoid extinction areas (and possible associated molecular gas emission). This procedure was followed as best as possible given the constraints of the Pool observing periods allocated to this project. Some signal in the OFF has been detected in some scans, but as the OFF positions are changing with time, on average it disappears in the reduction process. Some signal can be underestimated but this should be included in the global $20 \%$ calibration uncertainties. A total of 540 raw spectra were recorded in $21.5 \mathrm{~h}$ of telescope time with a spectral resolution of $2.6 \mathrm{~km} \mathrm{~s}^{-1}$. Table 1 displays the epoch of observations, for each day the average system temperature, the total integration time accounting for two independent polarization measurements, and the number of pixels and scans performed. We provide main-beam temperatures throughout this paper with beam and forward efficiencies: $B_{\text {eff }}=63$ and $F_{\text {eff }}=94$.

Each spectrum was first visually inspected to remove those affected by very unstable baselines. They were then processed to iteratively correct for the instrumental response: (1) a linear baseline was subtracted for each scan; (2) the scans were averaged for each position and smoothed; (3) spectra with a clear detection had a higher order polynomial subtracted to optimize the baseline subtraction. The typical $\mathrm{rms}$ in each final pixel was at $1 \sigma T_{\mathrm{mb}}=3.9 \mathrm{mK}$ in $13 \mathrm{~km} \mathrm{~s}^{-1}$ channels. Thirteen of the 36 positions exhibit a detection above $3 \sigma$ (as displayed in Fig. 1).

Upper limit on the continuum. We estimated the value of the continuum at $1 \mathrm{~mm}$ in the field M31-1a. Four percents of the spectra have a mean value outside the range $[-0.1,0.1] \mathrm{mK}$ and were removed. The remaining spectra are averaged and smoothed over the whole bandwidth (936 Mhz). We find a continuum level of $0.041 \pm 0.043 \mathrm{mK}$. We thus derive a $3 \sigma$ upper limit of $0.13 \mathrm{mK}$ on the continuum emission, corresponding to $S_{\text {continuum }}<0.65 \mathrm{mJy}$.

\section{Analysis}

\subsection{Molecular data}

Table 2 summarizes the characteristics of the $\mathrm{CO}(2-1)$ lines detected in this field. A Gaussian function was fitted to each line to determine its area $I_{\mathrm{CO}}$, central velocity $V_{0}$, width $\sigma$ and peak temperature $T_{\text {peak }}$. The baseline rms is provided for each line. We assumed a standard Galactic $X_{\mathrm{CO}}=N_{\mathrm{H}_{2}} / I_{\mathrm{CO}}=$ $2.3 \times 10^{20} \mathrm{~cm}^{-2}\left(\mathrm{~K} \mathrm{~km} \mathrm{~s}^{-1}\right)^{-1}$ following Strong et al. (1988). However, note that different values have been adopted in the literature. Leroy et al. (2011), relying on the Nieten et al. (2006) $\mathrm{CO}$ data with a strong signal $\left(I_{\mathrm{CO}}>1 \mathrm{~K} \mathrm{~km} \mathrm{~s}^{-1}\right)$, estimated a lower value $X_{\mathrm{CO}}=9.66 \pm 1.33 \times 10^{-19} \mathrm{~cm}^{-2}\left(\mathrm{~K} \mathrm{~km} \mathrm{~s}^{-1}\right)^{-1}$ for the inner part of M 31 .

Relying on the $\mathrm{CO}(2-1) / \mathrm{CO}(1-0)$ line ratios measured by Melchior \& Combes (2011) in this area, we assumed a line ratio of 1 and thus adopt the previous $X_{\mathrm{CO}}$ ratio for the $\mathrm{CO}(2-1)$ line. We then converted the $N_{\mathrm{H}_{2}}$ column density to an $\mathrm{H}_{2}$ mass surface density and derived a molecular mass $M_{\text {beam }}$ assuming the gas fills the main beam. Lastly, when all the positions were averaged a noise level of $0.8 \mathrm{mK}$ was achieved, but no signal appeared. This stacking demonstrates that there is no extended emission higher than $2.4 \mathrm{mK}$.

One third of the observed positions exhibit a $\mathrm{CO}(2-1)$ detection, as displayed in Fig. 1. Apart from the spectra 18 and 23 (and the second component of spectra 36), all the detected lines are redshifted with respect to the systemic velocity.

\subsection{Dust extinction and dust emission}

In this area devoid of large amounts of gas (e.g. Loinard et al. 1996), we have shown in Melchior \& Combes (2011) that in the northwestern part of the bulge of M31, CO is detected where extinction is observed, while it is not detected in areas where no extinction is measured. This supports the dust-gas correlation observed in the Milky Way (Bohlin et al. 1978) and other galaxies (e.g. Foyle et al. 2012). Smith et al. (2012) also claimed that the gas in M 31 is well-traced by dust at a constant metallicity. The optical and near-infrared data displayed in Fig. 2 provide complementary information to our $\mathrm{CO}$ detection. The left panel displays the observed extinction as computed in Melchior et al. (2000). The bulge light is mostly dominant within $R<1.2 \mathrm{kpc}$ $(\sim 300$ arcsec) from the center (Courteau et al. 2011), where $R$ is the projected distance to the center on the sky plane. We modeled its photometry with elliptical annuli using the standard surface 
Table 2. Characteristics of the $\mathrm{CO}(2-1)$ lines.

\begin{tabular}{|c|c|c|c|c|c|c|c|c|c|c|}
\hline \# & Offsets & $R\left({ }^{\prime \prime}\right)$ & $\begin{array}{c}I_{\mathrm{CO}}\left(\mathrm{K} \mathrm{km} \mathrm{s}^{-1}\right)= \\
\int T_{\mathrm{mb}} \mathrm{d} V\end{array}$ & $V_{0}\left(\mathrm{~km} \mathrm{~s}^{-1}\right)$ & $\sigma\left(\mathrm{km} \mathrm{s}^{-1}\right)$ & $\begin{array}{l}T_{\mathrm{mb}}^{\text {peak }} \\
(\mathrm{mK})\end{array}$ & $\begin{array}{l}\text { Baseline } \\
\text { rms (mK) }\end{array}$ & $N_{\mathrm{H}_{2}}\left(\mathrm{~cm}^{-2}\right)$ & $\begin{array}{c}\Sigma_{\mathrm{H}_{2}} \\
\left(M_{\odot} \mathrm{pc}^{-2}\right)\end{array}$ & $M_{\text {beam }}\left(M_{\odot}\right)$ \\
\hline 5 & $-33.0,-8.33$ & 34.0 & $0.51 \pm 0.12$ & $-301.7 \pm 4.0$ & $30.1 \pm 8.6$ & 16.0 & 4.2 & $1.17 \times 10^{20}$ & 1.99 & $3.25 \times 10^{3}$ \\
\hline 15 & $-9.0,-32.3$ & 33.5 & $0.60 \pm 0.08$ & $-248.3 \pm 1.7$ & $24.4 \pm 3.9$ & 23.0 & 3.2 & $1.38 \times 10^{20}$ & 2.35 & $3.84 \times 10^{3}$ \\
\hline 17 & $-9.0,-8.3$ & 12.2 & $0.76 \pm 0.13$ & $-137.4 \pm 3.9$ & $41.9 \pm 7.3$ & 17.1 & 4.0 & $1,75 \times 10^{20}$ & 2.97 & $4.85 \times 10^{3}$ \\
\hline 17 & $-9.0,-8.3$ & 12.2 & $0.26 \pm 0.08$ & $-32.7 \pm 3.9$ & $13.3 \pm 29.1$ & 18.4 & 4.0 & $0.60 \times 10^{20}$ & 1.02 & $1.67 \times 10^{3}$ \\
\hline 18 & $-9.0,-3.7$ & 9.7 & $0.35 \pm 0.09$ & $-355.0 \pm 4.1$ & $27.8 \pm 7.0$ & 11.7 & 3.5 & $0.81 \times 10^{20}$ & 1.37 & $2.24 \times 10^{3}$ \\
\hline 19 & $-3.0,-56.3$ & 56.4 & $0.13 \pm 0.05$ & $-341.4 \pm 4.0$ & $13.1 \pm 3.9$ & 9.2 & 3.8 & $0.30 \times 10^{20}$ & 0.51 & $0.83 \times 10^{3}$ \\
\hline 19 & $-3.0,-56.3$ & 56.4 & $0.10 \pm 0.03$ & $-339.9 \pm 4.0$ & $7.5 \pm 2.2$ & 12.9 & 5.1 & $0.23 \times 10^{20}$ & 0.39 & $0.64 \times 10^{3}$ \\
\hline 21 & $3.0,-32.3$ & 32.4 & $0.50 \pm 0.13$ & $-224.0 \pm 6.9$ & $48.1 \pm 11.1$ & 9.8 & 3.9 & $1.15 \times 10^{20}$ & 1.96 & $3.20 \times 10^{3}$ \\
\hline 22 & $3.0,-20.3$ & 20.5 & $0.20 \pm 0.07$ & $-67.1 \pm 4.0$ & $17.2 \pm 6.2$ & 11.0 & 3.0 & $0.46 \times 10^{20}$ & 0.78 & $1.27 \times 10^{3}$ \\
\hline 23 & $3.0,-8.3$ & 8.8 & $0.34 \pm 0.09$ & $-390.4 \pm 4.0$ & $29.5 \pm 8.1$ & 10.9 & 3.2 & $0.78 \times 10^{20}$ & 1.33 & $2.17 \times 10^{3}$ \\
\hline 24 & $3.0,3.7$ & 4.8 & $0.33 \pm 0.09$ & $-256.6 \pm 5.0$ & $28.6 \pm 8.6$ & 11.0 & 3.1 & $0.76 \times 10^{20}$ & 1.29 & $2.11 \times 10^{3}$ \\
\hline 26 & $15.0,-44.3$ & 46.8 & $0.48 \pm 0.14$ & $-207.8 \pm 5.7$ & $39.8 \pm 14.5$ & 11.2 & 4.1 & $1.10 \times 10^{20}$ & 1.88 & $3.07 \times 10^{3}$ \\
\hline 26 & $15.0,-44.3$ & 46.8 & $0.45 \pm 0.16$ & $-121.1 \pm 7.7$ & $47.0 \pm 21.9$ & 9.1 & 4.1 & $1.03 \times 10^{20}$ & 1.76 & $2.87 \times 10^{3}$ \\
\hline 28 & $15.0,-20.3$ & 25.2 & $0.36 \pm 0.08$ & $-190.9 \pm 1.0$ & $13.0 \pm 33.1$ & 26.1 & 3.8 & $0.83 \times 10^{20}$ & 1.41 & $2.30 \times 10^{3}$ \\
\hline 28 & $15.0,-20.3$ & 25.2 & $0.22 \pm 0.05$ & $-189.7 \pm 1.9$ & $14.1 \pm 3.1$ & 14.4 & 5.6 & $0.51 \times 10^{20}$ & 0.86 & $1.40 \times 10^{3}$ \\
\hline 35 & $27.0,-8.3$ & 28.2 & $0.15 \pm 0.05$ & $-160.9 \pm 3.3$ & $13.0 \pm 4.79$ & 10.9 & 3.6 & $0.35 \times 10^{20}$ & 0.59 & $0.96 \times 10^{3}$ \\
\hline 35 & $27.0,-8.3$ & 28.2 & $0.064 \pm 0.026$ & $-155.0 \pm 0.8$ & $3.4 \pm 1.8$ & 17.8 & 5.4 & $0.15 \times 10^{20}$ & 0.25 & $0.41 \times 10^{3}$ \\
\hline 36 & $27.0,3.7$ & 27.3 & $0.94 \pm 0.16$ & $-152.8 \pm 4.6$ & $52.6 \pm 10.6$ & 16.8 & 4.1 & $2.16 \times 10^{20}$ & 3.68 & $6.01 \times 10^{3}$ \\
\hline 36 & $27.0,3.7$ & 27.3 & $0.43 \pm 0.10$ & $-315.6 \pm 2.8$ & $21.9 \pm 5.3$ & 18.3 & 4.1 & $0.99 \times 10^{20}$ & 1.68 & $2.74 \times 10^{3}$ \\
\hline
\end{tabular}

Notes. Spectra are smoothed to a $13 \mathrm{~km} \mathrm{~s}^{-1}$ resolution, but the fits in italic have been performed on $2.6 \mathrm{~km} \mathrm{~s}^{-1}$ spectra. The offsets refer to the center of the M31-1a field.

photometry algorithm developed for IRAF (Jedrzejewski 1987). This model is intended to reproduce the light profile along the bulge of M 31 without extinction. The median intensity over the elliptical annulus sectors was used to avoid areas that suffer extinction. Large-scale extinction following the elliptical profile could affect this model, but this is obviously weak. The observed extinction is defined as $A_{\lambda}=-2.5 \log _{10}\left(\phi_{\text {obs }} / \phi_{\text {model }}\right)$, where $\phi_{\text {obs }}$ is the observed brightness and $\phi_{\text {model }}$ the modeled brightness. This provides a lower bound for the true internal extinction, with the following assumptions: (1) the gas lies in front of the bulge and; (2) there is only one clump of gas per line of sight. This automatically eliminates large-scale homogeneous extinction like the one caused by the Milky Way. On the one hand, as the resolution of the optical data is close to 1 arcsec, corresponding to $3.8 \mathrm{pc}$ at the distance of M31 (the optical resolution is a factor of 10 better than that achieved with $\mathrm{CO}$ observations), the second assumption is reasonable for most lines of sight given the small masses detected. According to the mass-size relation for molecular clouds first observed by Solomon et al. (1987), we expect that the more massive molecular clouds detected here have a size smaller than 3 pc (e.g. Lombardi et al. 2010). On the other hand, it is most probable that not all the gas lies in front of the bulge in the southern part. The observed extinction corresponds to $A_{\text {observed }}=-2.5 \log _{10}[f+(1-f) \times \exp (-\tau)]$, where $f$ is the fraction of light in front of the dust and $\tau$ the real optical depth at a given wavelength. In principle, if several clumps were present along the line of sight, we could decompose the extinction $A_{\text {observed }}$ into several components. In practice, more observational constraints would be necessary to perform such a detailed analysis. It is important to note that the majority of the dust and associated gas is not diluted in the whole bulge and not spread all along the line of sight. It is expected to be relaxed and to lie in a disk-like structure (or ring).

The top panel of Fig. 3 displays how the observed extinction relates to the fraction $f$ of light in front of the dust for different values of real internal extinction. For extinction values above 5 , it is very difficult to separate the real value of the extinction from optical observations. For a given value of observed extinction, it is, however, possible to put some constraint on the positions of dust clumps. Relying on the modeling of Tempel et al. (2011), we estimated how the fraction $f$ of light in front of the dust relates to the position $z$ of the dust clump along the line of sight. The bottom panels of Fig. 3 display this relation for different lines of sight along the minor axis $Y$ (projected on the sky plane). If the line of sight crosses the center (where there is a cusp due to the stellar cluster), the fraction $f$ is varying strongly because most of the light comes from an area smaller than $1 \operatorname{arcsec}(\sim 3.8 \mathrm{pc})$. For lines of sight varying from $10 \mathrm{pc}$ to $1 \mathrm{kpc}$, the slope of $f(z)$ is decreasing. There are two main effects to be stressed. (1) The observed extinction is very sensitive to the location of the dust clump along the line of sight. In the central region, dust just behind the mid-plane could easily escape optical detection because the fraction of light in front of the clump can be very significant. (2) This effect is additionally strengthened by the asymmetry caused by the inclination: the far side will have a higher percentage of light than the near side.

The real extinction is difficult to measure from optical data, but the observed extinction provides some constraints on the location of the dust along the line of sight, as displayed in Fig. 2. For instance, if $A_{\text {observed }}=0.2$, the real extinction is higher than 0.25 and the fraction of light $f$ in front of the dust is between 0.2 and 0.85 . Accordingly, as a function of the chosen line of sight, it is possible to constrain the position of the dust clump. For a position at $100 \mathrm{pc}$ (resp. $10 \mathrm{pc}$ ) from the center, the dust clump is expected to lie within $400 \mathrm{pc}$ (resp. $150 \mathrm{pc}$ ) from the plane perpendicular to the line of sight passing through the center. Note that these observations have a resolution of $12 \operatorname{arcsec}(\sim 45 \mathrm{pc})$, so the gas situated very close (in projected distance) to the black hole could lie anywhere between 0 and $150 \mathrm{pc}$ on the far side.

The right panel of Fig. 2 displays the dust emission at $8 \mu \mathrm{m}$. It is not affected by extinction, but depends on dust grains and their heating. The southern part exhibits a much stronger dustemission intensity than the observed extinction, suggesting that the dust clumps lie on the far side. 

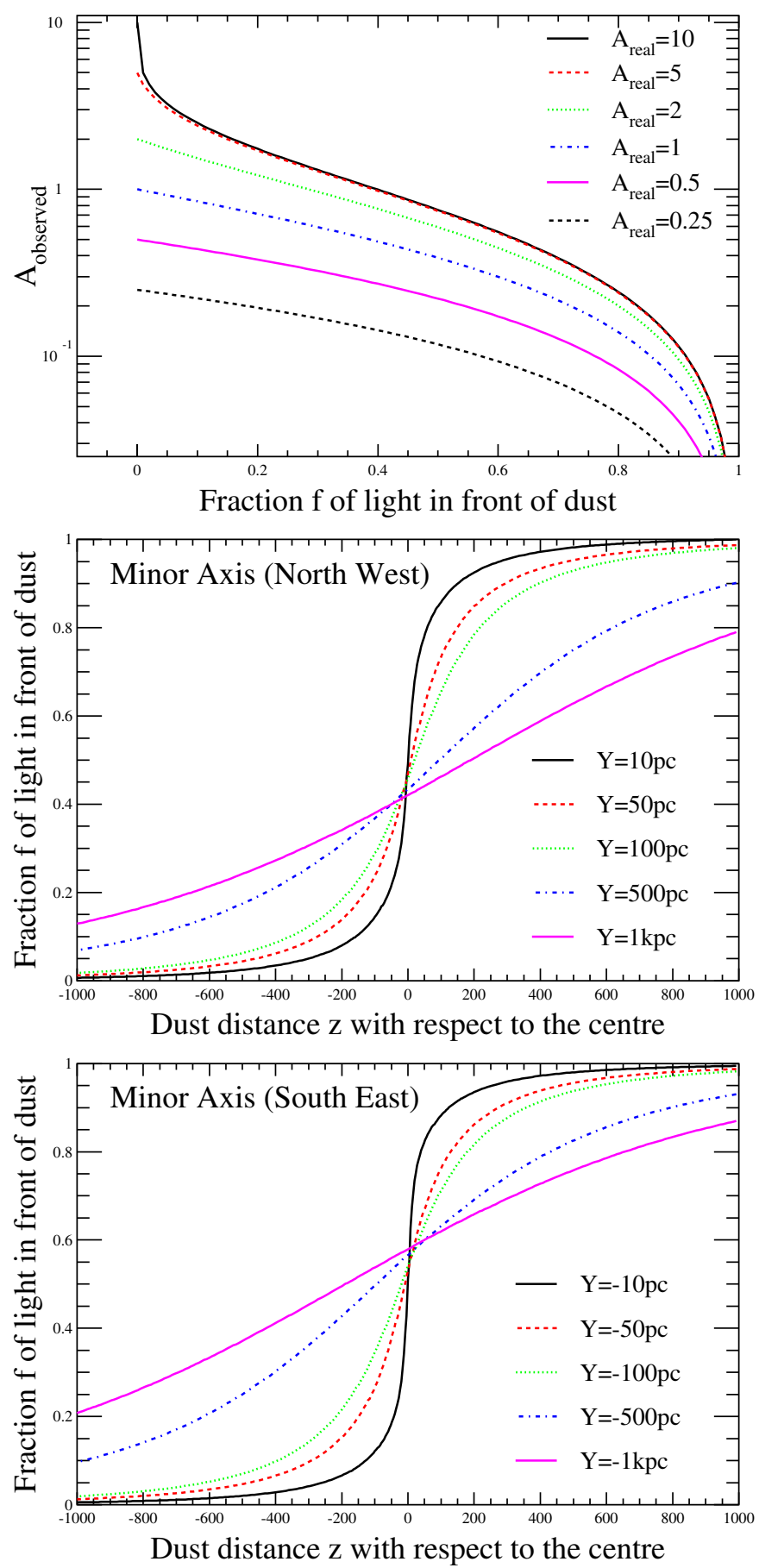

Fig. 3. Relationship between extinction and the position of the dust clumps along the line of sight. The upper panel displays how the observed extinction $A_{\text {observed }}$ relates to the fraction $f$ of light in front of the dust for different values of real extinction $A_{\text {real }}$. The lower panels display how the fraction $f$ relates to the line of sight distance $z$ with respect to the center for different projected distances $Y$ to the center along the minor axis. We detect a near/far side asymmetry, which strengthens the effect of absence of observed extinction on the far side. We rely on the modeling of Tempel et al. (2011).

\subsection{Characteristics of the dust components}

Following the previous discussion on the expected near/far asymmetry expected due to the inclination of M31, one can note that Ciardullo et al. (1989) first detected an asymmetry between the near and far sides among the bulge's planetary nebulae
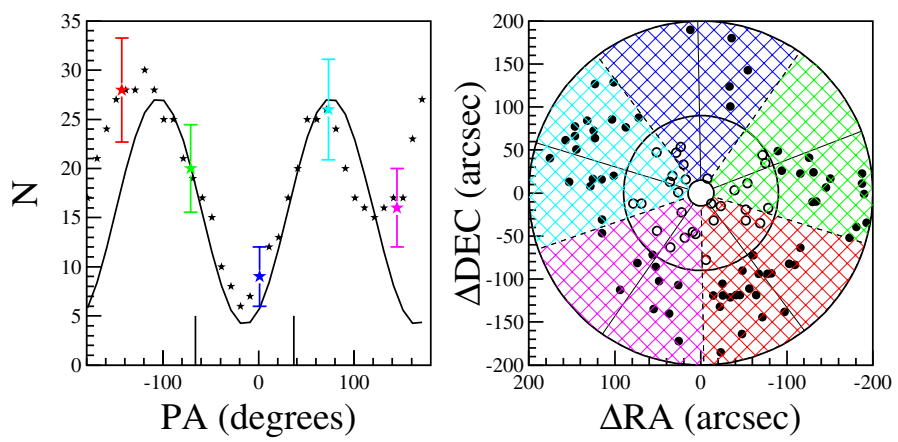

Fig. 4. Two dust components that affect the Ciardullo et al. (1989) sample of planetary nebulae. We considered a complete sample of 99 planetary nebulae distributed into a 15-200 arcsec annulus. This annulus was split into five equal parts as indicated by the colors. The left panel displays the relation between the number $N$ of planetary nebulae detected in each position angle. The color star points (in the left panel) correspond to the colored hatched areas displayed in the right panel. The black stars (in the left panel) were obtained with a running sum for different position angles (PA). The error bars indicated only for the color points correspond to Poisson statistical noise. The line corresponds to the multiplication of two sinusoids of phase PA $=37 \mathrm{deg}$ and $-66 \mathrm{deg}$, as indicated by the ticks at the bottom. The right panel displays the spatial distribution of the planetary nebula sample in the annuli, corresponding to the completeness limits (15, 90 and 200 arcsec).

distribution. A similar geometrical effect is also expected in the distribution of microlensing events in M 31's bulge (e.g Kerins et al. 2001, 2006). An asymmetry between the near side and the far side, caused by extinction in the main plane, is observed in the distribution of planetary nebulae in the $10 \mathrm{kpc}$ ring (Merrett et al. 2006).

We reinvestigated the catalog of planetary nebulae of Ciardullo et al. (1989), which samples the bulge area quite well. There is a complete sample of 99 planetary nebulae: it is spatially complete and Ciardullo et al. (1989) computed the detection efficiencies with respect to the surface magnitude. As displayed in the right panel of Fig. 4, 29 are present in a 15-90 arcsec annulus within a m5007 magnitude smaller than 22.1, and 70 in a 90-200 arcsec annulus with a m5007 magnitude lower than 22.7. This region within 200 arcsec from the center is dominated by the bulge, and the planetary nebulae follow the light distribution. In the left panel of Fig. 4, we counted the number of planetary nebulae in five parts of the 15-200 arcsec annulus and displayed them as a function of the position angle. The five points follow a sinusoid. The star points (not independent) were obtained similarly with a running sum for intermediate position angles.

According to the near/far side asymmetry, one would expect a sinusoidal variation of the number of planetary nebulae with a $2 \pi$ period with respect to the position angle of the main disk $37 \mathrm{deg}$. Surprisingly, the observed period is $\pi$. The overplotted sinusoid is varying as $\sin (\mathrm{PA}-37) \times \sin (\mathrm{PA}+66)$, which is compatible with the superposition of two dust components whose main axis have respective position angles of 37 degrees and -66 degrees. This is a new confirmation of the presence of two gas/dust components in this region: the main disk (and mainly the $10 \mathrm{kpc}$ ring) seen in projection with a PA of 37 degree, and the inner ring seen in the infrared (e.g. with Spitzer data) with a position angle of -66 degree. The amplitude of the effect is similar for both components. There is probably an additional perturbation (at the limit of detection) close to PA $=-180 / 180 \mathrm{deg}$, which might correspond to a noncircular structure. More statistics are required to be more conclusive. 

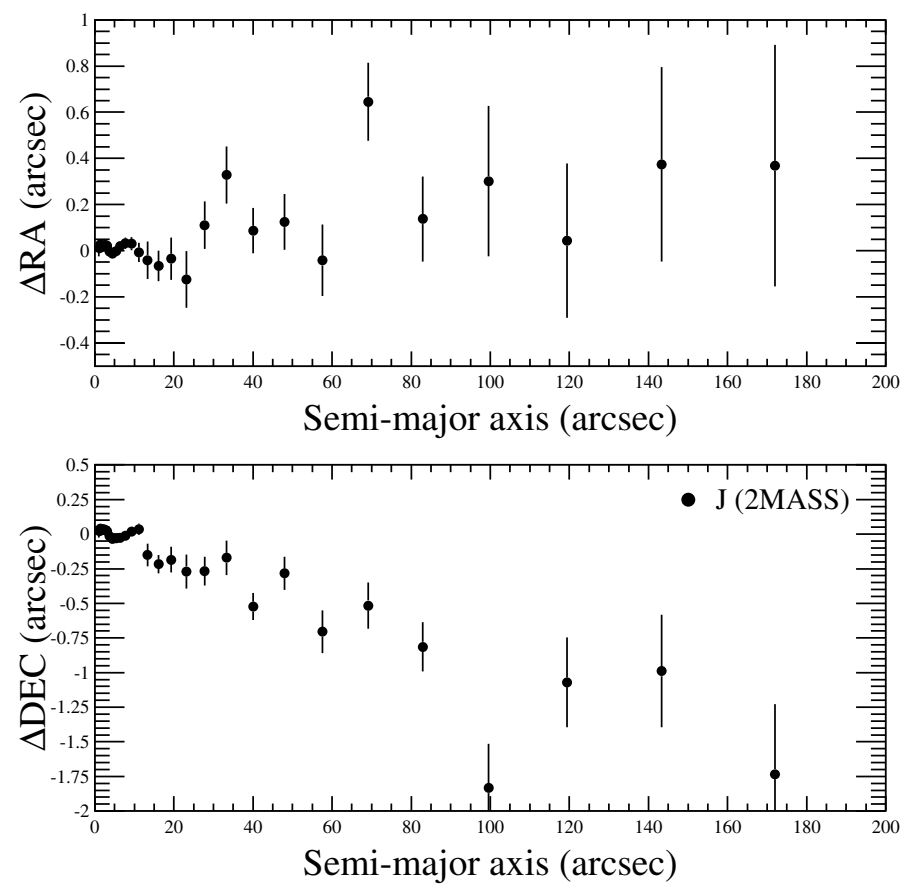

Fig. 5. Variations of the position of the centers of the elliptical annuli computed on the 2MASS $J$ image as a function of the semi-major axis.

Those two dust components expected to be associated with $\mathrm{CO}$ detections have an orientation compatible with our previous discussion: the clumps detected in $\mathrm{CO}$ are most probably on the farside. In addition, one could argue that the few points detected close to the systemic velocity (and blue shifted) could be associated to the main disk (possibly at large scale).

\subsection{Ionized gas}

The middle panel of Fig. 2 shows the $\mathrm{H} \alpha$ and [NII] emission map (Ciardullo et al. 1988). As discussed by Rubin \& Ford (1971), it is dominated by [NII] excited by shocks. The overall pattern corresponds to the $8 \mu \mathrm{m}$ map, but there is no exact correspondence. (1) These wavelengths are affected by extinction. For instance, the position observed in CO by Melchior et al. (2000) (double circle in Fig. 2) seems to be affected by extinction. (2) The kinematics of the [NII] line measured by Boulesteix et al. (1987) (see also Melchior \& Combes 2011) exhibit a disk in rotation and the $\left(40^{\prime \prime} \times 40^{\prime \prime}\right)$ circumnuclear region is blueshifted with respect to the systemic velocity. In parallel, the velocity field measured in $\mathrm{CO}$ is redshifted. It is thus probable that both components are decoupled.

This can be related to the compilation of all gas velocities in the inner 10 arcmin of M 31 by Stark \& Binney (1994). The isovelocity curves are very irregular and chaotic, even involving much larger scales than here.

\subsection{Bulge light emission}

The bulge model computed on photometric images as described in Sect. 3.2 provides the ellipse geometry parameters (see e.g. Kent 1989). We performed this modeling on 2MASS $J$ data ${ }^{1}$ (Skrutskie et al. 2006) and Fig. 5 displays the position of the centers of each annulus computed. In contrast with $B$ and

\footnotetext{
1 We chose the $J$ image because it offers a better signal-to-noise ratio than the $K$ image.
}

$\mathrm{H} \alpha /[\mathrm{NII}]$ data, we do not expect any bias due to dust obscuration. Interestingly, the center of the annuli is systematically shifting toward the south by about $7 \mathrm{pc}$ within $0.78 \mathrm{kpc}$. It is tempting to compare this off-centering of the bulge with the off-centering $(\sim 350 \mathrm{pc})$ of the inner dust ring detected by Block et al. (2006). Under the hypothesis of a coupled $m=1$ motion between the inner bulge and the disk, and given the Tamm et al. (2012) estimation of the mass of M31's bulge of about $4 \times 10^{10} M_{\odot}$, we can expect a mass of gas in the inner ring of $9 \times 10^{8} M_{\odot}$, if the maximum of light corresponds to the barycenter.

As discussed in Appendix A and shown in Fig. A.1, the bulge is triaxial but we do not expect our results to be affected as the amplitude of the twist is about $10 \mathrm{deg}$.

\section{Interpretation}

As displayed in Fig. 2, some detections (7/13) correlate well with the Spitzer dust emission and several positions ${ }^{2}$ (5, 17, 18, 19, 23, and 26) do not. None of the positions detected in CO correspond to any observed $A_{\text {observed }}^{B}$ extinction. According to the available kinematics, it is most probable that the ionized component is decoupled from the molecular gas. The observed extinction is lower than 0.025 in $B$ (see left panel of Fig. 2). Relying on Fig. 3, we can derive that the typical percentage $f$ of light in front of the average clump is higher than $90 \%$. Following the modeling of Tempel et al. (2011), the average clump lies at a depth between 20 and $200 \mathrm{pc}$ from the center on the far side, depending on its projected distance. It could be farther away if the real extinction is significantly higher than 0.25. Accordingly, the positions without Spitzer infrared emission could be much farther away where the radiation field is too weak to heat the dust, except within $4 \mathrm{pc}$ of the black hole, where the light of the nuclear star cluster prevents the detection of any extinction.

The kinematics is complex and do not exhibit a clear pattern. Most of the lines are redshifted with respect to the systemic velocity, while four are blueshifted. This is surprising as the opposite trend was observed in this field by Melchior \& Combes (2011) with the optical ionized gas. The gas detected at the northeast side (Melchior et al. 2000) was also redshifted, so this is not simply a counter-rotation. In addition, the velocity range is spread from -33 to $-390 \mathrm{~km} \mathrm{~s}^{-1}$.

These results are compatible with the $0.7 \mathrm{kpc}$ inner ring scenario discussed in Melchior \& Combes (2011) that was initially proposed by Block et al. (2006): the ring is tilted, which could explain why the gas is here on the far side and the velocities are redshifted. It lies off-center, which could explain why we do not see a regular rotation pattern, since we are far from the kinematical center. As supported by the dust components detected with the planetary nebulae in Sect. 3.3, the $0.7-\mathrm{kpc}$ inner ring is most probably superimposed on the 10 -kpc ring in the main disk: this could account for the multiple velocity components together with the clumpiness.

In summary, we have shown the presence of molecular gas close to the black hole. There is no extended diffuse molecular emission, but we have detected small dense clumps located on the far side of the bulge. These are located between $20 \mathrm{pc}$ and $215 \mathrm{pc}$ in projected distance from the center (and observed with a resolution of $45 \mathrm{pc}$ ). Assuming a single dust/gas clump per line of sight and some modeling assumptions, we showed that clumps corresponding to these lines of sight lie on the far

\footnotetext{
2 The Spitzer map seems to exhibit a defect close to the center at the position of spectrum 17 .
} 
A.-L. Melchior and F. Combes: A cold-gas reservoir to fuel the M31 nuclear black hole and stellar cluster
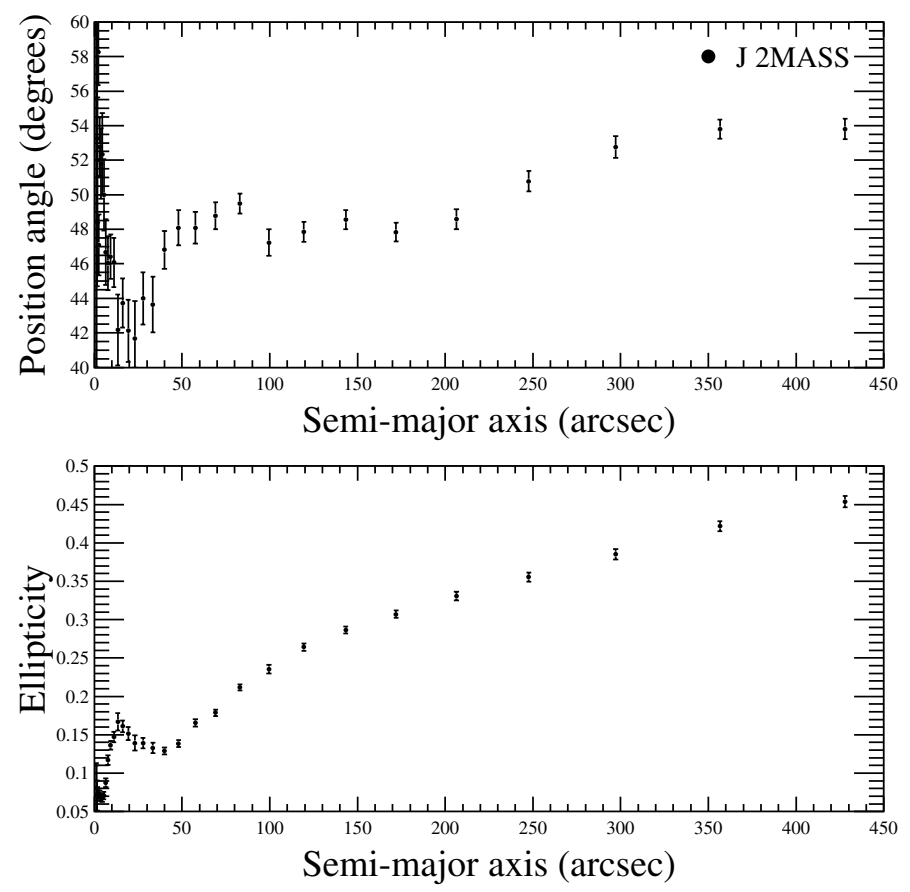

Fig. A.1. Position angles and ellipticities of the centers of the elliptical annuli computed on the 2MASS $J$ image as a function of the semi-major axis. These points correspond to the centers of the annuli presented in Fig. 5.

side, at least at $150 \mathrm{pc}$ in depth from the center and most probably closer than $600 \mathrm{pc}$. If some gas is present next to the center, it is also most probably on the far side but could be very close to the black hole. The kinematics exhibit an unexpected trend: most of the lines are redshifted with respect to the systemic velocity, which could be due to the off-centering. Along several lines of sight, there are multiple CO components. Our reanalysis of the Ciardullo et al. (1989) catalog of planetary nebulae revealed two components of position angles $37 \mathrm{deg}$ and $-66 \mathrm{deg}$. In the light of our molecular detections discussed here, we claim that dust and gas from the outer $10 \mathrm{kpc}$ ring are superimposed on the inner $0.7 \mathrm{kpc}$ ring in this very central region. The inclination of both components is such that the gas and dust in the southern area lie mainly on the far side.

Acknowledgements. This article is based on observations (067-11, 221-11) performed in the IRAM-30 m Pool session at Pico Veleta (Spain). We are most grateful to Manuel Gonzalez, who monitored this program through the IRAM$30 \mathrm{~m}$ Pool observing sessions. We also thank the IRAM staff at Pico Veleta for excellent support at the telescope. IRAM is supported by INSU/CNRS (France), MPG (Germany) and IGN (Spain). We thank the anonymous referee for his/her constructive comments. David Valls-Gabaud is warmly acknowledged for his helpful comments. This publication makes use of data products from the Two Micron All Sky Survey, which is a joint project of the University of Massachusetts and the Infrared Processing and Analysis Center/California Institute of Technology, funded by the National Aeronautics and Space Administration and the National Science Foundation.

\section{Appendix A: Triaxality of the bulge}

Figure A.1 displays the variation of the position angles and ellipticities computed in the modeling described in Sect. 3.2. There is a clear isophot twist that was not caused by extinction. The most plausible explanation is the triaxiality of the bulge as discussed by Kormendy (e.g. 1982). Our values are similar to those presented by Beaton et al. (2007), but they are presented here in linear scale in accordance with Fig. 5.

The isophot twist is significant but its amplitude does not exceed $10 \mathrm{deg}$ and should not affect our results significantly. (1) The bins used to compute the position angle of the dust component from the planetary nebulae distribution are $72 \mathrm{deg}$. (2) This triaxiality could affect the near/far side effect, but it should be a second-order effect.

\section{References}

Azimlu, M., Marciniak, R., \& Barmby, P. 2011, AJ, 142, 139

Bacon, R., Emsellem, E., Combes, F., et al. 2001, A\&A, 371, 409

Bajaja, E., \& Shane, W. W. 1982, A\&AS, 49, 745

Barmby, P., Ashby, M. L. N., Bianchi, L., et al. 2006, ApJ, 650, L45

Beaton, R., Majewski, S., Patterson, R., et al. 2007, BAAS, 39, 104.17

Bender, R., Kormendy, J., Bower, G., et al. 2005, ApJ, 631, 280

Block, D. L., Bournaud, F., Combes, F., et al. 2006, Nature, 443, 832

Bogdán, Á., \& Gilfanov, M. 2008, MNRAS, 388, 56

Bohlin, R. C., Savage, B. D., \& Drake, J. F. 1978, ApJ, 224, 132

Boulesteix, J., Georgelin, Y. P., Lecoarer, E., Marcelin, M., \& Monnet, G. 1987, A\&A, 178, 91

Braun, R., Thilker, D. A., Walterbos, R. A. M., \& Corbelli, E. 2009, ApJ, 695, 937

Chemin, L., Carignan, C., \& Foster, T. 2009, ApJ, 705, 1395

Ciardullo, R., Rubin, V. C., Ford, W. K., Jr., Jacoby, G. H., \& Ford, H. C. 1988, AJ, 95, 438

Ciardullo, R., Jacoby, G. H., Ford, H. C., \& Neill, J. D. 1989, ApJ, 339, 53

Courteau, S., Widrow, L. M., McDonald, M., et al. 2011, ApJ, 739, 20

del Burgo, C., Mediavilla, E., \& Arribas, S. 2000, ApJ, 540, 741

Dressler, A. 1984, ApJ, 286, 97

Emerson, D. T. 1976, MNRAS, 176, 321

Foyle, K., Wilson, C. D., Mentuch, E., et al. 2012, MNRAS, 421, 2917

Garcia, M. R., Hextall, R., Baganoff, F. K., et al. 2010, ApJ, 710, 755

Gillessen, S., Genzel, R., Fritz, T. K., et al. 2012, Nature, 481, 51

Groves, B., Krause, O., Sandstrom, K., et al. 2012, MNRAS, 426, 892

Jacoby, G. H., Ford, H., \& Ciardullo, R. 1985, ApJ, 290, 136

Jedrzejewski, R. I. 1987, MNRAS, 226, 747

Kent, S. M. 1989, AJ, 97, 1614

Kerins, E., Carr, B. J., Evans, N. W., et al. 2001, MNRAS, 323, 13

Kerins, E., Darnley, M. J., Duke, J. P., et al. 2006, MNRAS, 365, 1099

Kormendy, J. 1982, Saas-Fee Advanced Course 12: Morphology and Dynamics of Galaxies, 113

Kormendy, J., \& Bender, R. 1999, ApJ, 522, 772

Lauer, T. R., Faber, S. M., Groth, E. J., et al. 1993, AJ, 106, 1436

Lauer, T. R., Bender, R., Kormendy, J., Rosenfield, P., \& Green, R. F. 2012, ApJ, 745,121

Leroy, A. K., Bolatto, A., Gordon, K., et al. 2011, ApJ, 737, 12

Li, Z., Wang, Q. D., \& Wakker, B. P. 2009, MNRAS, 397, 148

Li, Z., Garcia, M. R., Forman, W. R., et al. 2011, ApJ, 728, 10

Loinard, L., Allen, R. J., \& Lequeux, J. 1996, A\&A, 310, 93

Lombardi, M., Alves, J., \& Lada, C. J. 2010, A\&A, 519, L7

Melchior, A.-L., \& Combes, F. 2011, A\&A, 536, 52

Melchior, A.-L., Viallefond, F., Guélin, M., \& Neininger, N. 2000, MNRAS, 312,29

Merrett, H. R., Merrifield, M. R., Douglas, N. G., et al. 2006, MNRAS, 369, 120

Nieten, C., Neininger, N., Guélin, M., et al. 2006, A\&A, 453, 459

Riess, A. G., Fliri, J., \& Valls-Gabaud, D. 2012, ApJ, 745, 156

Rubin, V. C., \& Ford, W. K., Jr. 1971, ApJ, 170, 25

Saglia, R. P., Fabricius, M., Bender, R., et al. 2010, A\&A, 509, A61

Schuster, K.-F., Boucher, C., Brunswig, W., et al. 2004, A\&A, 423, 1171

Skrutskie, M. F., Cutri, R. M., Stiening, R., et al. 2006, AJ, 131, 1163

Smith, M. W. L., Eales, S. A., Gomez, H. L., et al. 2012, ApJ, 756, 40

Stark, A. A., \& Binney, J. 1994, ApJ, 426, 31

Solomon, P. M., Rivolo, A. R., Barrett, J., \& Yahil, A. 1987, ApJ, 319, 730

Strong, A. W., Bloemen, J. B. G. M., Dame, T. M., et al. 1988, A\&A, 207, 1

Tabatabaei, F. S., \& Berkhuijsen, E. M. 2010, A\&A, 517, A77

Tamm, A., Tempel, E., Tenjes, P., Tihhonova, O., \& Tuvikene, T. 2012, A\&A, 546, A4

Tempel, E., Tuvikene, T., Tamm, A., \& Tenjes, P. 2011, A\&A, 526, A155

Vilardell, F., Ribas, I., \& Jordi, C. 2006, A\&A, 459, 321 